\title{
Hacia una Investigación Educativa abierta y social: más allá del modelo ANECA
}

\section{Towards an open and social Educational Research: beyond the ANECA model}

\author{
Enrique Javier Díez Gutiérrez
}

Recibido: 20 de noviembre de 2019 Aceptado: 5 de mayo de 2020 Publicado: 31 de julio de 2020

To cite this article: Díez-Gutiérrez, E. J. (2020). Hacia una Investigación Educativa abierta y social: más allá del modelo ANECA. Márgenes, Revista de Educación de la Universidad de Málaga, 1 (2), 3-18

DOI: http://dx.doi.org/10.24310/mgnmar.v1i2.7185

\section{RESUMEN}

La investigación educativa está cada vez más presionada por el modelo de evaluación establecido por la ANECA. "Publicar o perecer" en revistas de impacto se ha convertido en la única vía para poder acceder o mantenerse en la "carrera investigadora", lo cual genera un modelo de ciencia y de investigación marcado por el impacto y la rentabilidad, frente al bien común y el interés social que se ven postergados progresivamente. Se necesita voluntad política para impulsar otro modelo de evaluación de la investigación comprensivo, razonable, global, equitativo, coherente y justo, que responda a un modelo de Ciencia Abierta y Ciudadana para el bien común, mandato expreso del programa de la Unión Europea en su iniciativa de Horizonte 2020.

Palabras clave: investigación educativa; capitalismo académico; ciencia abierta; ciencia ciudadana

\section{ABSTRACT}

Educational research is increasingly pressured by the evaluation model established by ANECA. "Publish or perish" in impact journals has become the only way to access or stay in the "research career." This generates a model of science and research marked by impact and profitability, against the common good and social interest that are progressively delayed. Political will is needed to promote another comprehensive, reasonable, global, equitable, coherent and fair research evaluation model that responds to an Open and Citizen Science model for the common good, express mandate of the European Union program in the Horizon 2020 initiative.

Keywords: educational research; academic capitalism; open science; citizen science 


\section{ESTUDIOS Y E N SAYOS}

\section{INTRODUCCIÓN}

Más de 3.000 investigadores y científicos sociales de universidades y organismos de investigación de toda España, así como de Francia, Portugal, Reino Unido, Estados Unidos, Brasil o Suecia, denunciaron en un manifiesto en noviembre de 2019 (AA.VV., 2019), con motivo de las elecciones en España, que los dirigentes del partido fascista español Vox, de reciente aparición en el escenario político hispano, han construido "una parte importante de su argumentario político a través del falseamiento y la manipulación de datos e informaciones diversas”, que implican la criminalización de los inmigrantes o la negación de la violencia de género. Esta estrategia del partido ultraderechista de "revestir con datos supuestamente objetivos y contrastados lo que es simplemente una agenda ideológica de nacionalismo extremo basado en la intolerancia, el racismo y la xenofobia”, afirma el manifiesto, no solo deteriora "las bases de nuestra convivencia mediante el recurso insistente a la mentira y la distorsión de los datos existentes” sino que, además, “atenta, desprestigia y tergiversa el trabajo de miles de investigadores sociales”.

Este manifiesto sumó 2.000 adhesiones en menos de 24 horas, y superaba las 3.000 el 10 de noviembre, día de las elecciones generales en España, solo tres días después de que se hubiera creado e iniciado la recogida de firmas, a través de un formulario online, lo cual nos indica su rápida difusión y la gran acogida y apoyo que tuvo por la comunidad científica.

Esta implicación y posicionamiento de una parte de la comunidad científica social, y entre ellos los y las investigadoras educativas, en determinados momentos de "emergencia" (auge del fascismo en España y su discurso de odio basado en datos falseados, en este caso), contrasta con el ensimismamiento o incluso solipsismo habitual del que suele hacer gala buena parte de la comunidad científica, salvo honrosas excepciones o pequeños grupos muy reducidos, más activistas, respecto a cuestiones sociales y políticas. La mayoría parece no querer o no tener tiempo para centrarse en tareas de investigación y divulgación que no sean las estrictamente exigidas por las instituciones o agencias de acreditación de las que dependen para su evaluación y progreso en la carrera académica e investigadora.

Enfrascados muchos de ellos, presionados por sus “carreras” y la promoción necesaria para asentarse y conseguir un puesto relativamente estable, en un contexto universitario e investigador cada vez más precario y temporal ${ }^{1} \mathrm{y}$, por lo tanto, cada vez más competitivo, las estrategias se centran sobre todo en esa promoción y, solo posteriormente o en segundo término, luchar por cambiar las condiciones que han generado esta situación.

\section{2. “DISCIPLINAR” A QUIEN INVESTIGA}

Dice el adagio “o vives como piensas, o acabas pensando como vives”. La agotadora carrera académica e investigadora va consumiendo el tiempo, las fuerzas, las esperanzas y las ganas de lu-

\footnotetext{
1 Un 42,4\% del personal investigador en la universidad pública española tiene contrato temporal, a pesar de que este porcentaje excede el límite máximo permitido por ley, un 40\%. Aunque hay comunidades autónomas que sobrepasan ampliamente ese límite, como las Islas Baleares (57,9\% de temporales) o Catalunya (54,3\%). Los campus tienen un 4\% menos de profesorado que hace seis años y se ha invertido la tendencia por la que el personal funcionario con contratos estables eran mayoría: ahora lo son los contratados con empleo precario, temporal e inestable. Donde había profesores titulares funcionarios, ahora hay asociados en precario (Sánchez Caballero, 2017).
} 


\section{EST U D I O Y E N SAYOS}

cha y de cambio de una situación de precariedad, presión continua y autoexplotación desmedida (Goyanes, \& Rodríguez-Gómez, 2018). Ante lo cual buena parte se van aclimatando y termina por considerar como inamovible el modelo y la única solución posible: adaptarse lo mejor posible a él (Van-Dalen, \& Henkens, 2012). Es el mandato principal del modelo neoliberal: aprender a asumir la realidad que hay, y a adaptarse a ella para aprovecharla en tu beneficio, porque "no hay alternativa". El mantra que popularizó Margaret Thatcher y que convirtió en lema neoliberal (George, \& Wolf, 2002). Es el denominado “pensamiento pragmático”, que estimula el individualismo y la búsqueda de soluciones personales frente a las luchas colectivas.

Aderezado con la denominada "ciencia de la felicidad" o "pensamiento positivo" (Carr, 2007), donde la crisis, la explotación y la precariedad se convierte en una oportunidad para demostrar su aptitud, su valía y de lo que se trata es de afrontarlo cambiando la actitud, no las condiciones de explotación y precariedad que afectan a todos y todas (Bejar, 2015). Manuales de autoayuda para reforzar el yo y adaptarse a la realidad. Cada cual debe aprender a creer que se trata de utilizar los recursos que se encuentran en el interior de uno mismo para tener éxito. Esto supone, en definitiva, aprender a autocupabilizarse, porque el único responsable de lo que a cada uno le sucede es uno mismo (Fernández-Río, \& Vilariño, 2016).

Esta ideología de la automotivación se convierte en una técnica de sumisión, haciendo la tarea que en siglos pasados desempeñaba el capataz en las fábricas (Díez, 2018), pero en este caso con nuestra aceptación y consentimiento; incluso deseando ser parte del sistema (Han, 2014), en donde parece que cada día es el último y se debe luchar como si no hubiera mañana, sin tiempo siquiera para descansar, charlar reposadamente con los colegas, en una situación de asfixia permanente, estando siempre ocupado en la próxima investigación, en el siguiente paper, congreso o comunicación (Hernández, Nepomiachi \& Ré, 2017). Eso sí, cobrando como un becario, o incluso pagando por trabajar y adquirir experiencia laboral ${ }^{2}$. Puestos de trabajo precarios y temporales, rayando el sueldo mínimo interprofesional pero, por supuesto, acompañados del "salario emocional" de "estar en la universidad”, "tener prestigio", todo ello revestido de toda una narrativa épica, que se ha convertido en casi un género literario (Cantó, 2017; López Menacho, 2018, 2019).

Este modelo ANECA, de publicar o perecer, se ha convertido en un dispositivo de control y sumisión que presiona a quien investiga a “conducirse” bajo la presión de la competición, en función del cálculo del máximo interés individual. Esta competición encarna una "nueva éti-

2 Estos días he participado en una concentración en la Universidad pública contra la explotación laboral de estudiantes como mano de obra, ya ni siquiera barata, sino gratuita. Es la nueva semiesclavitud del capitalismo uberizado que se instala incluso en las universidades públicas como consecuencia de los recortes en su financiación y la gestión de las mismas como si fueran empresas, avanzando hacia el modelo de "ubersidad" precarizada. El sindicato estudiantil convocante de la concentración denunciaba que el alumnado universitario está realizando "trabajo gratuito disfrazado de formación y experiencia”, para suplir la "falta de personal" en el Hospital Veterinario de la Universidad. Pero lo que más me ha sorprendido ha sido la reacción de algunos de los alumnos y alumnas que obtuvieron una de estas plazas. Me acerqué a hablar y conocer su opinión, pues estaban situados en frente de quienes participábamos en la concentración y denunciábamos esta explotación. Me contestaron que, tras haberse reunido con la dirección del Hospital y la Facultad de Veterinaria, lo tenían claro, afirmando que "todos nosotros hemos solicitado participar en este programa de prácticas extracurriculares de forma voluntaria, sin percibirlo en forma alguna como una explotación". Así lo han publicado en un comunicado que sale difundido en algunos medios, junto a las declaraciones de la dirección. Alegando que "así se hace en el resto de Facultades de Veterinaria de España" y que son "actividades totalmente voluntarias". Esto es lo que me ha sorprendido, sobre todo: la defensa que los propios explotados hacen de su explotación. La "libertad de elegir" ser explotados. 


\section{ESTUDIOS Y E N SAYOS}

ca”, que los procedimientos de evaluación de la ANECA y de las instituciones universitarias e investigadoras se encargan de reforzar (Laval \& Dardot 2013), para volverse cada vez más eficaz en conseguir resultados, medibles y cuantificables, principalmente en publicaciones que “anecables”. La finalidad es que este nuevo sujeto considere que el éxito, que incluso autorrealizarse, es incrementar su esfuerzo por ser lo más competitivo posible, como si ese afán surgiera de su interior, de su propio deseo. La autoexplotación se convierte así en disciplina personal (Han, 2012). A partir de entonces somos los únicos responsables de lo que nos sucede, pues ya no hay condiciones o problemas sociales, sino nuestra actitud ante ellos. Las luchas sociales se convierten en competiciones individuales. Los derechos colectivos se abandonan en pos de carreras personales.

Además, este solipsismo al que nos avoca el actual modelo de competición constante en la carrera investigadora, que responde al modelo neoliberal individualista, que nos asegura que ha sido por "libre elección", genera unas tasas de sufrimiento personal que están por encima de la media $^{3}$ (Lozano, 2019).

\section{3. “DISCIPLINAR” LA INVESTIGACIÓN}

En un contexto neoliberal como en el que se halla inmerso la academia, gestionadas las universidades como si fueran empresas (Brunner et al., 2019), concebida la investigación fundamentalmente al servicio de la expansión económica del capital, la prioridad se ha convertido en lo explotable, lo comercializable, sean las patentes, las 'spin off' o la conversión de la investigación en activos rentables para el mundo empresarial y la producción tecnológica (Cantoral, 2007). Esto supone, no solo que la industria no invierta en investigación que no sea rentable a corto plazo, lo cual afecta de lleno a la investigación educativa (Aguado-López, \& Becerril-García, 2016), sino que incluso las instituciones y administraciones públicas no la incluyan entre sus prioridades (no tenemos más que revisar las líneas RIS3 de I+D establecidas para optar a financiación pública).

Es más, lo que se acaba es introduciendo el control de la gran empresa privada sobre la investigación (Lee, 2014), mediante la financiación de congresos e investigaciones, algo habitual en multinacionales sanitarias, petroleras, tabaqueras o alimentarias (Castañeda, 2017). Una de las últimas investigaciones sobre el tema (Rey-López \& González, 2018, 813), publicada en la revista de la Asociación Europea de Salud Pública, demuestra que 8 millones de euros destinados por la multinacional Coca-Cola a financiar estudios científicos "sirvieron a sus intereses comerciales y, en muchos casos, no concuerdan con los esfuerzos para mejorar la salud de la población”.

Este control privado de la investigación pública es también alentado por voluntad política de determinados partidos políticos de orientación neoliberal o conservadora. Por ejemplo, las convocatorias de financiación de I+D en la Comunidad Autónoma de Castilla y León, en

3 Una investigación desarrollada en Bélgica (Levecque et al., 2017), refleja que casi la mitad de los doctorandos sufrían síntomas de sobrecarga, tristeza continua, depresión, pérdida de sueño, pérdida de confianza, sentimiento de inutilidad o imposibilidad de tomar decisiones. Otro estudio de la Royal Society (Guthrie, 2017) encontró que más del 40\% de investigadores doctorales y postdoctorales, que son quienes llevan el peso de las investigaciones, presentaban síntomas de depresión o problemas relacionados con niveles altos de estrés. Otras investigaciones obtienen resultados similares (Evans et al., 2018; Goyanes, \& Rodríguez-Cómez, 2018; Van-Dalen, \& Henkens, 2012). 


\section{ESTUDIOS Y E N SAYOS}

España, imponen la obligatoriedad de tener contratos o convenios con empresas privadas en el momento en el que se solicite ayuda o subvención pública, condicionando la financiación pública a la previa obtención de financiación privada. Lo cual puede pervertir el sentido y la finalidad de la investigación, dado que la investigación que no tiene inmediato rendimiento mercantil, es decir, de la que pueden obtenerse patentes o resultados comercializables, no parece suscitar mucho interés para el modelo empresarial predominante, que presume de buscar el beneficio rápido e inmediato y no una visión a largo plazo sobre un tipo de investigación de alto valor añadido o que beneficie a la comunidad. Pero también porque ese requisito condiciona la financiación.

Este enfoque impide de hecho investigaciones fundamentales relacionadas con temas regionales y locales de interés, sobre todo relacionados o que afectan a la gente empobrecida (Gates, 2002), las minorías o las mujeres de clase trabajadora, las zonas rurales, etc. ¿Qué empresa está dispuesta a financiar una investigación sobre la educación pública, o sobre la integración escolar del alumnado extranjero, si no puede rentabilizar y 'monetizar' sus resultados? Por lo que nos encontramos con que aquellos proyectos de investigación que buscan analizar y solucionar problemas relevantes para los sectores de la comunidad que más necesitados están no encuentran suficiente financiación pública.

Si a esto sumamos que se está decidiendo desde gobiernos tanto conservadores como neoliberales las líneas prioritarias de investigación, que ahora denominan de "especialización inteligente" (estrategias RIS3), estableciendo que "solamente tendrán financiación pública aquellos proyectos de investigación en estos ámbitos determinados”, la consecuencia es que está excluyendo la investigación en ciencia básica, así como en ciencias jurídicas, económicas y sociales además de humanidades. El resultado es que, en los últimos cuatro años, han sido excluidos el $80 \%$ de los proyectos presentados en Castilla y León (Último Cero, 2016), muchos de ellos relacionados con la Educación, la Historia, el Patrimonio, la Comunicación, la Literatura, el Derecho, la Economía, la Arqueología o la Química inorgánica.

De esta forma se está disciplinando a la investigación y a los investigadores e investigadoras para que se adapten al modelo, para que piensen y diseñen sus investigaciones de acuerdo a este modelo de rentabilidad, de comercialización, de utilidad económica. El valor de cambio se impone sobre el valor de uso. Y su explotación comercial se impone sobre su utilidad social. Las humanidades, las artes, los saberes críticos han perdido la batalla.

De hecho, las mismas universidades valoran los resultados de las investigaciones si esto les permite obtener posiciones sobresalientes en rankings universitarios. Esta transformación sigue lógicas globales, promovidas por organizaciones internacionales de carácter económico (FMI, OMC, OCDE, etc.), que se han instalado en la agenda pública, hasta el punto que se asumen como propias y, por lo tanto, legítimas (Pineda, \& Celis, 2017). De tal forma que la propia universidad se está transformando, pasando de un lugar para realizar trabajo académico, investigar y debatir sobre la ciencia a ritmo pausado y profundo, a convertirse en una gran maquinaria, un potente negocio, una gran empresa competitiva que requiere la obtención de resultados cuantificables, que puedan ser patentados, transferidos y explotados y que procu- 


\section{ESTUDIOS Y ENSAYOS}

ren una buena colocación en los índices de productividad investigadora y permitan estar en la parte alta del ranking de Shanghái, del CSIC o de cualquier otro que aparezca en los medios de comunicación.

Incluso se están transformando los criterios de financiación de las universidades y los institutos de investigación, que ya no se hace en función de las necesidades de la institución, sino de acuerdo a los resultados obtenidos en rankings estandarizados o en función de contratos definidos por la consecución de objetivos relacionados con esos rankings. Por eso, cada vez se recor$\tan ^{4}$ más los presupuestos para proyectos de orientación humanística y/o crítica, que se acaban considerando "improductivos" y se avala lo que se percibe desde la institución como económicamente útil (Apple, 2000).

\section{4. “DISCIPLINAR” LA DIFUSIÓN DE LA INVESTIGACIÓN}

El “disciplinamiento" de la investigación se ve agravado por el "disciplinamiento" de la difusión de la misma (Goyanes, \& Rodríguez-Gómez, 2018). Porque esta difusión de la investigación educativa se ha reducido fundamentalmente a publicar artículos en determinadas revistas (Lee, 2014). Es lo que ya se denomina, entre la comunidad académica e investigadora, de forma irónica y a la vez sarcástica, "la loca carrera investigadora” (Jorge \& De Frutos, 2016-2017) o se describe con el mordaz aforismo coloquial de "publica o perece” (Cantoral, 2007; Aguado-López, \& Becerril-García, 2016).

Este enfoque se debe claramente a una decisión política. Han sido las políticas que la Administración Educativa española ha tomado sobre el sistema de evaluación y acreditación para acceder a un puesto docente e investigador en el ámbito universitario y para promocionar en la carrera académica, el que ha generado esta orientación. Por eso no es de extrañar que buena parte de los investigadores o investigadoras, sobre todo quienes están en situación precaria y necesitan conseguir "méritos" para estabilizarse en su carrera docente e investigadora, lo primero que tienden a preguntar, es: ¿pero, esto cuenta para la ANECA?, ¿esto me supondrá un “mérito” en la acreditación?, ¿esto servirá para el próximo sexenio que tengo que conseguir?, etc.

Porque el sistema de acceso, evaluación y consolidación de la carrera profesional universitaria e investigadora, especialmente a raíz del Real Decreto-ley 14/2012, premia especialmente la acumulación de publicaciones en revistas de impacto, que son las que supuestamente generan “distinción y reconocimiento" (acceso a una plaza en la universidad, consolidación en un puesto más estable, acumulación de sexenios por los que se cobra un plus, posibilidad de acceso a cuerpos superiores...), al tiempo que "penaliza" si no se consiguen (Reig, 2014). Si no se acumulan de forma regular y sistemática ven dificultado el acceso a un puesto, o bien la no promoción a

4 Según los informes anuales de la Confederación de Sociedades Científicas de España (COSCE) estos recortes han sido significativos sobre todo desde el año 2008, con una media del 24,6\% y llegando al 35\% durante la crisis, perdiendo 20.000 empleos en el sector de la investigación desde que se inició la crisis, porque España "es el país que más recortes ha realizado en este campo" (Molero \& de No, 2015; Corral, 2016; Mediavilla, 2017). Mientras, en plena crisis, la inversión en investigación en Alemania ha crecido un 38\% y la de Reino Unido, un 39\% (Sampedro, 2017). 


\section{EST U D I O Y E N SAYOS}

puestos superiores, pero también son penalizados con la reducción del salario al no percibir el plus correspondiente y, además, con un aumento significativo de lo que ahora se ha acabado

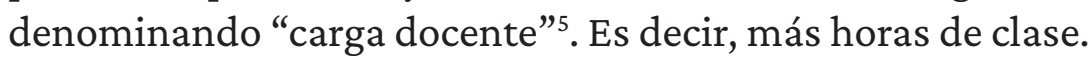

Para acceder a un puesto de profesorado universitario y, una vez que se accede, periódicamente cada seis años, la labor investigadora de todo el profesorado universitario de España, y de buena parte de los países a nivel mundial, es valorada en función de unos "índices de impacto" de publicaciones, controlados por un duopolio privado, gestionado por dos multinacionales que han convertido esto en un ingente negocio (Reig, 2014) a costa del dinero público ${ }^{6}$.

El actual sistema de acceso, valoración y acreditación de la carrera profesional universitaria se centra en valorar la publicación en determinadas revistas "de impacto" (Barbosa, 2019). Parece como si el artículo científico, que recibe muchas citas (impacto), fuera la única manera en que la ciencia se ha de presentar al mundo. Como si la ciencia solo contara si se ha publicado (Buranyi, 2017).

Claro, además hay que hacerlo en la revista con el mayor impacto ${ }^{7}$ posible, es decir, que reciba gran número de citas por parte de otros artículos. Ya no se trata de qué se ha descubierto con la investigación, ni qué se aporta a la ciencia con los resultados obtenidos, sino en qué revista se consigue publicar dicha investigación. De esta manera el número de citas recibidas en otros artículos, es decir, el factor de impacto, define realmente el valor científico e incluso su calidad (Martínez, \& Dorta, 2018). Publicar en una revista de "alto impacto" no es lo mismo que hacerlo en una que no está en las bases de datos del servicio Web of Science (WOS de la multinacional Clarivate Analytics) o Scopus, (perteneciente a la otra multinacional, Elsevier). Estas multinacionales controlan con mano férrea la calificación prestigiosa de revistas de "alto impacto", donde todo el mundo quiere publicar, porque son las que cuentan para ser realmente valorado y reconocido. Se creó así la "moneda” de prestigio en el mundo científico (Jorge \& De Frutos, 2016-2017), que estas dos multinacionales han convertido en un jugoso y lucrativo negocio.

Estos rankings de publicaciones han "universalizado" un modo de producción de conocimiento y de financiación de la investigación ligado a la academia estadounidense, con un efecto profundamente neocolonial, en donde el inglés se ha convertido en la lingua franca por excelencia (Beigel, 2018). Revistas cuya gran mayoría pertenece a países anglosajones, sobre todo Estados Unidos y Gran Bretaña, cuyos autores y autoras tienden a citar a sus compatriotas (Flores \& Villaseñor, 2015), dejando de lado especialmente aquellos que no son anglófonos. Generando de

5 Lo cual ha generado una desvaloración de la docencia que se la acaba considerando de menor valor, dado que además la evaluación positiva de la labor docente (los denominados quinquenios) cuentan de manera residual cuando se tienen en cuenta. Por lo que la finalidad fundamental de las universidades, la docencia en la educación superior, pasa a verse casi como una "carga docente", donde se "recarga" con más horas a quien no consigue la valoración positiva del sexenio (Rodríguez Victoriano, 2017). Cuando sabemos que no está probado -más bien al contrario- que haya una clara correlación entre productividad investigadora y docencia de calidad.

6 El negocio de la publicación científica genera unos ingresos globales superiores a los 22.000 millones de euros, cerca de lo que embolsa la industria del cine (Buranyi, 2017).

7 El llamado "factor de impacto" mide la importancia de una revista, en base a la frecuencia en la que los artículos son citados por otros, es decir, la cantidad de citas en otros artículos que reciben los artículos que se publican en ella. Por cierto, al margen la naturaleza positiva o negativa de dichas citas. 


\section{EST U D I O Y E N SAYOS}

forma colateral una idea de "ciencia periférica", vinculada a la ciencia desarrollada en los espacios alejados de este circuito 'principal' (Barbosa, 2019). Centradas en análisis y metodologías que interesan a los anglosajones con relación a su realidad, pero poco permeables a estudios con otros modelos o enfoques. Para poder publicar en estas revistas es obligado referenciar un tipo de literatura muy alejada de la realidad no anglosajona renunciando a objetos, marcos teóricos y metodologías que serían los más apropiados para el análisis de la propia realidad y contexto social donde se desarrolla la labor investigadora (Barsky, 2014; Ramió, 2014).

Este sistema, que se ha impuesto como forma de evaluación de la carrera profesional universitaria también en los países de esos circuitos de "ciencia periférica", impuesto por las políticas de las Administraciones Educativas, sustituyeron los instrumentos de evaluación docente que universidades españolas o latinoamericanas, por ejemplo, en el ejercicio de su autonomía, ya realizaban (Amigot \& Martinez, 2015).

En realidad, podemos decir que este sistema importa, de manera totalmente acrítica y literal, un modelo de productividad del mundo empresarial, convirtiendo la ciencia así "en una mercancía: está en el mercado y se paga un precio por ella” (Mirowski, 2011; Monbiot, 2017; Saltelli, 2017).

Pero la actual política de los gobiernos sigue empeñada en empujar a la comunidad académica a la publicación en revistas incluidas en los índices de Clarivate Analytics -a través de su producto Web of Knowledge- y Elsevier -a través de su producto Scopus- para promocionar en la carrera universitaria. Ha sido, por tanto, decisión política de los gobiernos el que se haya supeditado la evaluación a los datos del factor de impacto ofrecidos por dos multinacionales, al establecer que se computen como "méritos" de investigación prácticamente solo las publicaciones realizadas en estas revistas, que son cuantificadas y “comercializadas” en esos índices de impacto controlados por las dos multinacionales, que cobran, solo en España, 5,5 millones anuales del erario público por el uso de sus bases de datos, y una de ellas, Elsevier ${ }^{8}$, ingresa más de 24 millones al año de las universidades públicas por suscripciones a sus publicaciones (Buranyi, 2017; Munárriz, 2017; Villareal \& Escudero, 2018).

El negocio de este oligopolio multinacional es redondo: Los científicos y científicas trabajan, investigan y obtienen resultados bajo su propia dirección, con financiación en gran medida pública. Sus resultados se los ofrecen gratuitamente, sin ningún coste, a las revistas de estas multinacionales. Incluso en algunos casos tienen que pagar por ello. Bien de sus recursos propios o de la financiación recibida que se detrae de las labores de investigación para "invertirlo" en estas multinacionales (Casati, Giunchiglia, \& Marchese, (2010). A su vez, una vez recibido el trabajo realizado por quienes investigan, y ajustado a los criterios y reglas que establecen las revistas de estas multinacionales, ellas se lo pasan a otros científicos y científicas para que revisen la validez científica y juzguen si el trabajo merece ser publicado, un proceso que conlleva mucho trabajo, conocido como revisión por pares, que lo tienen que hacer de forma voluntaria. Y, por último, estas multinacionales venden entonces el producto a quienes quieran acceder a él: bibliotecas públicas, de universidades, instituciones de investigación. Al final de la cadena quienes realizaron

8 En 2010 esta multinacional declaró 827 millones $€$ de beneficios en publicaciones científicas (36\% más de los que declararon Apple, Google o Amazon ese año). En 2012 y 2013 los beneficios Elsevier subieron al 40\% (Buranyi, 2017; Monbiot, 2017 ). En 2016 facturó 2.600 millones $€$. España ha pagado en los últimos cuatro años 98 millones de euros (Villareal \& Escudero, 2018). 


\section{EST U D I O Y E N SAYOS}

el producto tienen que pagar por leer su propia producción. El coste de un artículo va desde 31,51 dólares (si se quiere acceder a varios artículos, hay que pagar por cada uno) hasta una suscripción anual con un coste medio de 379 dólares (la suscripción a algunas revistas cuesta diez mil dólares al año o más). Es un sistema “de triple pago" (Monbiot, 2017), en el que "el Estado financia la mayor parte de la investigación, paga los salarios de la mayoría de los que revisan la calidad de la investigación, y luego compra la mayor parte del trabajo publicado. (...) Lo que aquí vemos es puro capitalismo rentista, monopolizar un recurso público y luego cargar un precio desorbitante para utilizarlo" (Buranyi, 2017). Como este mismo experto asegura: “en el futuro solamente quedarán un puñado de compañías editoriales inmensamente poderosas, y llevarán a cabo su actividad comercial en una era electrónica sin costes de impresión, convirtiéndose casi en puro beneficio”.

Todo ello, como decimos, en menoscabo de otros modelos públicos de evaluación en abierto que fueron sufriendo la política de recortes y la vinculación de las publicaciones en este sistema oligopolista a los factores de mayor impacto académico, así como de otros formatos y modos de valorar la carrera profesional por falta de voluntad política de las administraciones públicas. No solo las políticas públicas no se cuestionan el papel que desempeñan esos dos grandes holdings editoriales, sino que de forma indirecta están impidiendo que se valoren las publicaciones en repositorios públicos bajo fórmulas de acceso abierto, acordes con el mandato del artículo 37 de la Ley 14/2011, de la Ciencia, la Tecnología y la Innovación. No obstante, cuando hay voluntad política se puede iniciar el cambio de esta situación. Por ejemplo, en 2017, Alemania se plantó y se negaron a seguir pagando en los actuales términos, además de unir a sus 200 instituciones académicas para enfrentarse a estas multinacionales de forma conjunta y girar hacia un modelo de 'open access', que parece que les está funcionando (Villareal \& Escudero, 2018).

Lo más sorprendente de este sistema es que en buena parte de las ramas de conocimiento apenas nadie lee estas revistas y su importancia social tiende a ser escasa, exceptuando un número ínfimo (Guédon, 2011; Fischman, 2016). Pero lo cierto es que, en general, son revistas que en su mayor parte no tienden a cumplir con una función de difusión social a la ciudadanía (Gómez Méndez \& Sempere Serrano, 2017).

Se está presionando así a la comunidad científica para transformar la investigación rigurosa, que conlleva tiempo y procedimientos a veces lentos y reposados, hacia una "loca carrera" de la "cultura del impacto y de la citación" para conseguir acumular reconocimiento de "impacto" y así poder obtener el acceso o el puesto estable o esos preciados sexenios que faciliten promocionar en la competitiva carrera universitaria. "Se ha llegado a un punto en que, para muchos ámbitos cientificos y profesores, fuera de las revistas indexadas no hay vida” (Ramió, 2014, 117).

También se han convertido en un filtro para promocionar, para ocupar determinados cargos académicos, para formar parte de comisiones de evaluación y para obtener recursos financieros ${ }^{9}$.

\footnotetext{
9 Los sexenios, aparte de estar vinculados a una cierta "reputación científica", los han establecido como condición para poder formar parte de las comisiones de acreditación para el acceso a los cuerpos docentes universitarios, para poder formar parte de los comités asesores de la CNEAI, para poder dirigir tesis doctorales (lo cual no es precisamente irrelevante para la actividad del profesorado universitario) o para poder ser designado miembro de las comisiones para decidir en concursos de acceso a sus plazas. Gráficamente podría decirse que, en la actualidad, para ser miembro de pleno derecho de la comunidad científica, hay que tener tres sexenios (Rodríguez de Santiago, 2012).
} 


\section{ESTUDIOS Y E N SAYOS}

"El sistema de sexenios tiene un enorme poder simbólico entre los profesores de universidad y poseerlos equivale al número de estrellas que tienen los generales” (Ramió, 2014, 133).

Este sistema de evaluación y acreditación del profesorado universitario está provocando, en definitiva, que la práctica investigadora se convierta en una "inversión” para incrementar el propio currículum, perdiendo de vista o postergando la dimensión social y de búsqueda del bien común que debería tener toda investigación y todo proceso personal y colectivo de investigación, máxime en el campo de la investigación educativa. Esto está primando el valor de cambio de la ciencia, ligado a las exigencias del mercado, y se acaba entrando en una espiral donde se asume como "normal" y "habitual" el conocimiento propietario y remunerado, instalándose en el inconsciente colectivo de la comunidad investigadora una relación con el conocimiento instrumental, acelerada, regida por el corto plazo y los resultados cuantificables. De esta forma se va olvidando que la ciencia debe estar al servicio de la humanidad y de la mejora del mundo en el que convivimos, ligada a problemas y necesidades sociales que no siempre son valorados por el mercado. Los efectos no se han hecho esperar: vemos como progresivamente se pospone la investigación base, que va desapareciendo progresivamente la dimensión crítica de la investigación, que se posterga o disminuye sustancialmente la investigación implicada en la resolución concreta de problemas locales de las comunidades periféricas o más necesitadas o aquellas investigaciones que potencian el avance del bienestar social, no digamos ya la investigación de corte etnográfico que tiene como condición la participación de la comunidad en el desarrollo de las investigaciones (investigación-acción participativa). Pero tiene también otros efectos colaterales: se abandonan de facto publicar monografías o libros con conocimiento reflexivo y pedagógico de fondo, capaces de alcanzar al gran público, pues son considerados “méritos menores" porque puntúan menos en la ANECA, frente a los artículos en revistas anglosajonas con índices medibles de impacto.

Todo esto está generando un proceso de competición interna entre los y las investigadoras y los equipos de investigación que fragmenta y enfrenta a quienes deberían cooperar, naturalizando la competencia en vez de producir formas colaborativas de pensamiento e investigación (Martínez, \& Dorta, 2018). La comunidad científica se ve así dividida entre la necesidad de investigar con el mayor rigor y reposo posible, colaborando y compartiendo los resultados y los logros para mejorar la sociedad al servicio de quien debería estar la investigación o rendir cuantitativamente en la "cultura del impacto y de la citación” para conseguir ser valorado por las instituciones evaluadoras y poder promocionar entre las precarias figuras del profesorado universitario (Martínez, \& Dorta, 2018). En este contexto, la posibilidad de disenso es difícil, pues los y las investigadoras se ven obligadas a cumplir las "reglas del juego" si quieren aspirar, en un horizonte incierto y competitivo, a mantener su puesto de trabajo (Nogueira, 2018).

\section{OTRA INVESTIGACIÓN EDUCATIVA ES POSIBLE}

Es necesario otro sistema de evaluación de la producción científica del profesorado universitario público, sin intereses comerciales, sin criterios de beneficios empresariales de quien controla el sistema de producción científica, basado en la colaboración, el conocimiento compartido, el libre acceso a la producción científica. Es decir, un modelo realmente público y democrático. 


\section{ESTUDIOS Y E N SAYOS}

Creo imprescindible virar el rumbo y apostar por una investigación educativa y por una evaluación de la misma que ponga en valor otros elementos: el trabajo compartido, abierto y colaborativo, la investigación de base y a largo plazo, la honestidad científica, pero sobre todo el compromiso con toda la sociedad y también con las necesidades y sectores más desfavorecidos. Al servicio del bien común. Este tipo de investigación debería ser la que las políticas educativas españolas y europeas deberían privilegiar en su financiación y en los criterios de evaluación y promoción.

Para ello debemos cambiar las políticas educativas que han establecido este modelo de evaluación de la producción científica. Decisiones políticas que en 2013 paralizaban, por recortes presupuestarios, proyectos públicos nacionales que podían ser una alternativa a este monopolio consentido y amparado públicamente. Alternativas posibles como el sistema DICE (Difusión y Calidad Editorial de las Revistas Españolas de Humanidades y Ciencias Sociales y Jurídicas) desarrollado desde 2006 por el CSIC. Al estar integrado en una institución pública, DICE no necesitaba recaudar dinero de las revistas que estudiaba y catalogaba, ni tampoco directamente de quienes lo consultaban. Suerte parecida sufrieron otros proyectos públicos de prestigio como el de INRECS, INRECJ o el INRECH de la Universidad de Granada.

Por lo tanto, hemos de ser conscientes de que nuestras administraciones públicas, que deberían gestionar lo público con criterios vinculados al bien común, han sido cooperadores necesarios (en financiación y difusión) del monopolio privado de esas dos multinacionales extranjeras para el desarrollo de su sistema comercial de evaluación, que han impuesto en toda nuestra geografía física y mental. La ANECA, que acredita la capacidad del profesorado, y la CNEAI, que reconoce a través de esos sexenios la actividad investigadora realizada, son las responsables de este sistema, en detrimento de otros formatos y modos de canalizar la actividad investigadora.

Frente a ello, si queremos que la investigación educativa y toda la investigación rompa este monopolio de hecho, debemos optar políticamente por apostar por los repositorios públicos y gratuitos de "acceso abierto", mandato expreso del programa de la Unión Europea Horizonte 2020, en la línea que plantea la Declaración de San Francisco sobre Evaluación de la Investigación (DORA, 2012) y la Declaración de Berlín, administrados por universidades u organizaciones de investigación públicas, que son una valiosa infraestructura que podrían apoyar la transición a un sistema de evaluación, comunicación y difusión académica más colaborativa y eficiente.

Evitando, claro está, que se conviertan de nuevo, como pretenden estas multinacionales, en otra forma de control con precios abusivos por la tasa de procesamiento del artículo (Article Processing Charge o APC), para publicar en los rankings que son valorados por la ANECA, volviendo al mismo círculo vicioso. Aunque se argumente que esos costes se pueden incluir como gasto del proyecto de investigación, lo cierto es que seguimos con el mismo esquema de financiar con dinero público la difusión que debería ser gestionada públicamente sin coste añadido. Repito, lo que falta es voluntad política.

Por eso se han presentado ya varias Proposiciones No de Ley (PNLs) en el Parlamento Nacional ${ }^{10}$ para, por una parte, superar este sistema de evaluación de la producción científica de los

10 Aunque están en "stand by", sin que hayan llegado a debatirse, dado la situación de cambios de gobierno y elecciones que ha habido en los últimos tiempos en España. 


\section{EST U D I O Y E N SAYOS}

investigadores y las investigadoras, y, por otra, ahorrar al Estado una ingente cantidad de dinero que pagamos a estas multinacionales extranjeras, así como sentar las bases para que la propia academia dé a luz un nuevo sistema consensuado de evaluación público, gratuito y abierto de la investigación. Un sistema más justo, que responda a un modelo de ciencia para el bien común, basado en repositorios institucionales públicos y que apueste por la ciencia abierta y ciudadana (Caldera-Serrano, 2019).

Las ventajas de este modelo de ciencia abierta u Open Science son considerables (Arzberger et al. 2004; Piwowar, Day \& Fridsma, 2007; Ramasamy et al., 2008; Torres-Salinas, 2012): el rendimiento económico para optimizar el dinero invertido en generación de conocimiento científico, sobre todo de aquellas investigaciones financiadas con dinero público, partiendo del principio de que los "resultados" de la investigación financiada públicamente son patrimonio de la humanidad y deben estar accesibles y disponibles tan amplia y directamente como se pueda (Torres et al., 2011); poder llevar a cabo metaanálisis de la ciencia; aumentar la visibilidad y citación; permitir el acceso sin tener que pagar; facilita el trabajo cooperativo entre redes de investigación y el poder ser hecha con y para la sociedad. Pero también facilita y potencia una ciencia ciudadana (Serrano et al., 2014, 2017; Eitzel et al., 2017; Canepa \& De La Fuente, 2019) que puede contribuir así con su participación activa (O’Carroll, 2017), algo que en la investigación educativa es crucial y básico.

De hecho, la ciencia ciudadana ${ }^{11}$ en Europa corresponde a una de las cinco orientaciones estratégicas del nuevo Programa de Trabajo 2018-2020 de “Ciencia con y para la sociedad" (SwafS) en el programa Horizonte 2020 y comienza además a ser visto como una parte integral de la Investigación e Innovación Responsable (RRI) y la Ciencia Abierta (“Open Science”). Esto permite, además, la democratización de la ciencia.

Tendría que ser acompañado por una política institucional que priorice la evaluación de las publicaciones electrónicas de acceso abierto a efectos de carrera académica, a la vez que se mantienen estándares de rigor y práctica científica solvente (revisión por pares), en donde haya diversidad geográfica y no haya ningún cargo ni tasa para poder publicar. Se podría hacer, como se indicaba anteriormente, a través de repositorios de acceso abierto, administrados por universidades $\mathrm{u}$ organizaciones de investigación, con una política clara e institucional de acceso abierto, así como una estrategia económica, tecnológica y legal que sustente el proceso, dado que además el acceso abierto y disponibilidad gratuita es un mandato de la Unión Europea, como veíamos.

Este nuevo sistema de evaluación y acreditación comprensivo, razonable, global, equitativo, coherente y justo, debería, por tanto, evaluar la investigación por sus contenidos y méritos y no por el método y fuente de difusión, aprovechar las oportunidades de la publicación en línea y en abierto, y publicar y difundir la investigación bajo la licencia Creative Commons Public Domain Dedication (Caldera-Serrano, 2018).

11 La ciencia ciudadana en Europa está representada por las actividades que genera la "Asociación Europea de Ciencia Ciudadana (ECSA, https://ecsa.citizen-science.net/)" que es una organización sin ánimo de lucro. Desde sus orígenes en el año 2013, la ECSA ha pasado a ser de una red informal de educadores y educadoras cívicas a una red de referencia de iniciativas de ciencia ciudadana para Europa. Por eso muchas de las acciones y actividades de Ciencia Ciudadana en Europa tienen un alto componente educativo. En la plataforma EU-Citizen.Science (http://eu-citizen.science) se pueden encontrar guías y recursos para iniciar proyectos de ciencia ciudadana así como ejemplos de iniciativas existentes. En España, la fundación Ibercivis (http:// www.ibercivis.es/) ha liderado diferentes iniciativas en Ciencia Ciudadana. 


\section{ESTUDIOS Y ENSAYOS}

En síntesis, podríamos resumir la propuesta global en los siguientes diez puntos clave:

1. Un sistema público de evaluación y acreditación del profesorado universitario que suponga una valoración justa y equilibrada, que no privilegie la investigación (reducida a número de publicaciones) y que deje de considerar la docencia como "de segunda" e incluso un cierto “castigo”, desvalorizando así la función principal universitaria.

2. Un sistema público que responda a un modelo de ciencia para el bien común que no priorice solo un determinado tipo de conocimiento "rentable" y tenga en cuenta criterios relacionados con la relevancia investigadora, pero también el impacto y utilidad social de la labor investigadora.

3. Un sistema público apoyado en el modelo de ciencia abierta (en consonancia con el mandato europeo "Horizonte 2020"), que priorice la evaluación en publicaciones electrónicas de acceso abierto gratuito en la Internet pública, gratuitas tanto para publicar como para acceder a ellas, leerlas, descargarlas, etc. Eliminando o estableciendo la limitación y estandarización de tasas de publicación (APC), que, en todo caso, deben ser financiadas públicamente. Con el requisito de que quien publica conserve los derechos de autoría de su publicación bajo una licencia abierta como Creative Commons.

4. Un sistema público que no defina el valor y la "calidad" científica en función únicamente de la visibilidad de la producción, prescindiendo progresivamente de índices de impacto JCR y SJR.

5. Un sistema público que establezca un listado de revistas de alta calidad revisadas por pares, que sean en acceso abierto gratuito para publicar y acceder, especialmente en idioma español, a partir de los resultados de una encuesta de valoración al conjunto de la comunidad investigadora y docente, que sean las que se valoren en la publicación de la investigación de cada campo científico en España, en función de disciplinas, impulsando públicamente su mantenimiento y financiación si cumplen esos estándares. Promoviendo y apoyando económicamente una nueva generación de Publicaciones y Revistas de Acceso Abierto Gratuito, ayudando a las existentes para que opten por acometer la transición al acceso abierto gratuito.

6. Un sistema público que cree una plataforma a nivel nacional descentralizada de repositorios institucionales públicos abiertos cuyos contenidos sean admitidos por revisión de pares y que recoja la pluralidad de formatos y expresiones (libros, capítulos de monografías, soportes artísticos, software, etc.).

7. Un sistema público que no reduzca la valoración del trabajo investigador a artículos publicados y número de citas recibidas por esos artículos publicados, e incluya una valoración de calidad de los contenidos (valoración que aprecian los pares en las propuestas realizadas), así como la valoración en igual medida de trabajos y producciones científicas en todos los formatos y expresiones (libros, monografías, capítulos de monografías, revistas, informes, documentación, infografía, soportes artísticos, imagen, sonido, software, etc.).

8. Un sistema público implementado por el Ministerio de Ciencia y Universidades, en coordinación con las Comunidades Autónomas, a través de la Fundación Española para la Ciencia y la Tecnología (FECYT).

9. Un sistema que tenga asignado suficientes fondos e inversiones públicas para desarrollar esta alternativa, involucrar a la comunidad de autores y autoras, a los departamentos académicos en el apoyo a una transición al acceso abierto y gratuito (AAG) y comprometerse con las sociedades académicas y los consejos editoriales para apoyar un cambio al AAG. 


\section{EST U D O S Y E S A Y S}

10. Un sistema que sea negociado y consensuado con la comunidad académica de acuerdo a criterios comprensivos, razonables, globales, equitativos, coherentes y justos.

\section{REFERENCIAS}

AA.VV. (2019). Vox y las ciencias sociales. Manifiesto. [Recuperado de https://cutt.ly/ftmCwfL]

Aguado-López, E. y Becerril-García, A. (2016). ¿Publicar o perecer? El caso de las Ciencias Sociales y las Humanidades en Latinoamérica. Revista Española de Documentación Científica, 39(4), 151-168.

Amigot, P. y Martínez, L. (2015). Procesos de subjetivación en el contexto neoliberal. El caso de la evaluación del profesorado y la investigación universitaria. Revista de la Asociación de Sociología de la Educación, 8 (2), 138-155.

Apple, M. (2000). Teoría crítica y Educación. Madrid: Miño y Dávila Editores.

Arzberger, P.; Schoroedes, P.; Beaulieu, A.; Bowker, G.; Casey, K.; Laaksonen, L.; Moorman, D.; Uhlir, P. y Wouters, P. (2004). An international framework to promote Access to data. Science, 303 (5665), 1777-1778.

Barsky, O. (2014). La evaluación de la calidad académica en debate: los rankings internacionales de las universidades y el rol de las revistas cientificas. Buenos Aires, Argentina: Universidad Abierta Interarmericana-Teseo.

Beigel, F. (2018). Las relaciones de poder en la ciencia mundial. Un anti-ranking para conocer la ciencia producida en la periferia. Nueva Sociedad. [Recuperado de https://goo.gl/VQE7eh]

Brunner, J.J.; Vargas, J. R. L.; Ganga, F. y Rodríguez-Ponce, E. (2019). Idea moderna de universidad: de la torre de marfil al capitalismo académico. Educación XX1, 22(2), 119-140.

Buranyi, S. (2017). ¿Son los vertiginosos beneficios de la industria editorial malos para la ciencia? Sin Permiso. [Recuperado de https://goo.gl/zusCrg]

Calder-Serrano, J. (2018). Repositorios públicos frente a la mercantilización de la Ciencia: apostando por la ciencia abierta y la evaluación cualitativa. Métodos de Información, 9(17), 74-101. DOI: https://doi. org/10.5557/IIMEI9-N17-074101

Canepa, A. y de la Fuente, P. (2019). Ciencia Ciudadana: ciencia participativa como nueva herramienta docente. Primera Feria de la Educación Innovadora, Innova-Educa 2019. Burgos: Fundación Caja Burgos y Bit-Academy.

Cantó, P. (2017). Cinco nuevas palabras en inglés que nos quieren colar para que vivamos peor. $E l$ Confidencial. [Recuperado de https://goo.gl/BfrDqZ]

Cantoral, R. (2007). Publicar o perecer, o ¿publicar y perecer? Revista latinoamericana de investigación en matemática educativa, 10(3), 311-314.

Corral, M. (2016). España, el país europeo que más ha recortado en I+D durante la crisis. El Mundo, 10 de febrero de 2016. [Recuperado de https://goo.gl/sPrxcs]

DORA (2012). Declaration on research assessment. Declaración de San Francisco de evaluación de la Ciencia. Disponible en: http://blogs.ujaen.es/cienciabuja/wp-content/uploads/2013/10/dora.pdf

Eitzel, M.V., Cappadonna, J.L., Santos-Lang, C., Duerr, R.E., Virapongse, A., West, S.E., Kyba, C.C.M., Bowser, A., Cooper, C.B., Sforzi, A., Metcalfe, A.N., Harris, E.S., Thiel, M., Haklay, M., Ponciano, L., 


\section{ESTUDIOS Y ENSAYOS}

Roche, J., Ceccaroni, L., Shilling, F.M., Dörler, D., Heigl, F., Kiessling, T., Davis, B.Y. y Jiang, Q. (2017). Citizen Science Terminology Matters: Exploring Key Terms. Citizen Science: Theory and Practice, 2(1), p.1. DOI: http://doi.org/10.5334/cstp.96

Evans, T. M., Bira, L., Gastelum, J. B., Weiss, L. T. y Vanderford, N. L. (2018). Evidence for a mental health crisis in graduate education. Nature biotechnology, 36(3), 282.

Fischman, G. E. (2016). ¿Para qué y para quién investigamos?: estrategias de producción y de movilización del conocimiento de las facultades de educación en Norteamérica. [Recuperado de http://hdl.handle. net/10908/11737]

Flores, J.E. y Villaseñor, J.I. (2015). Perspectivas actuales sobre los rankings mundiales de universidades. Revista de Educación Superior, 44 (175), 44-67.

Gates, H. Jr (2002). Redifining the Relationship: The Urban University and The City in the 21st Century. Universities and Community Schools, 3, 17-22.

Gómez Méndez, L. Y Sempere Serrano, I. (2017). Preservar, difundir, comunicar: Del repositorio a las redes sociales ya la ciudadanía. RUIDERAe: Revista de Unidades de Información, 12, 1-11.

Guédon, J.C. (2011). El acceso abierto y la división entre ciencia "principal” y "periférica". Crítica y emancipación, 3(6), 135-180.

Guthrie, S., Lichten, C. A., Van Belle, J., Ball, S., Knack, A., y Hofman, J. (2017). Understanding mental health in the research environment. Santa Mónica, CA: RAND

Han, B. (2012). La sociedad del cansancio. Barcelona: Herder.

Hernández, S., Nepomiachi, E., y Ré, C. (2017). Seamos un país de 40 millones de emprendedores”. Interpretaciones ideológicas en tiempos neoliberales. Dossier de la Revista Ciencias Sociales, Facultad de Ciencias Sociales, Universidad de Buenos Aires, (93), 51-57.

Jorge, A. y De Frutos, R. (2016-2017). La evaluación de la investigación universitaria en España. Chasqui. Revista Latinoamericana de Comunicación, 133, 159-172.

Laval, Ch. Y Dardot, P. (2013). La nueva razón del mundo. Ensayo sobre la sociedad neoliberal. Barcelona: Gedisa.

Levecque, K., Anseel, F., De Beuckelaer, A., Van Der Heyden, J. y Gisle, L. (2017). Work organization and mental health problems in PhD students. Research Policy, 46(4), 868-879.

López-Menacho, J. (2018). Yo, Precario. Barcelona: Los Libros del Lince.

López-Menacho, J. (2019). La farsa de las startups. La cara oculta del mito emprendedor. Madrid: Catarata.

Mediavilla, D. (2017). El viejo truco del Gobierno para ocultar sus recortes en ciencia. El País, 5 de abril de 2017. [Recuperado de https://goo.gl/iJvgK]

Mirowski, Ph. (2011). Science-Mart. Privatizing American Science. Cambridge, MA: Harvard University Press.

Molero, J. y De No, J. (2015). Informe COSCE: Análisis de los recursos destinados a I+D+i (Política de Gasto 46) contenidos en los Presupuestos Generales del Estado Aprobados para el año 2015. [Recuperado de https:/L goo.gl/wGvW7M]

Monbiot, G. (2017). ¿Cómo nos metimos en este desastre? Madrid: Sexto Piso. 


\section{ESTUDIOS Y ENSAYOS}

Munárriz, A. (2017). Dos multinacionales controlan el negocio de los artículos científicos en España. Infolibre. [Recuperado de https://goo.gl/S5F53m]

Nogueira, A. (2018). En España, o investigas o 'anecas'. [Recuperado de https://goo.gl/YTqMSX]

O'carroll, C. (2017). Providing researchers with the skills and competencies they need to practise Open Science. Open Science Skills Working Group Report. Brussels: European Commission.

Pineda, P. y Celis, J. (2017). ¿Hacia la universidad corporativa? Reformas basadas en el mercado e isomorfismo institucional en Colombia. Archivos Analíticos de Políticas Educativas=Education Policy Analysis Archives, 25(1), 1-19.

Piwowar, H.A.; Day, R.S. y Fridsma, D.B. (2007). Sharing detailed research data in associated with increased citation rate. Plos one, 2 (3), e308. DOI: https://doi.org/10.1371/journal.pone.0000308

Ramasamy, A.; Mondry, A.; Holmes, C.C. y Altman, D.G. (2008). Key issues in conducting a metaanalysis of gene expression microarray datasets. Plos medicine, 5 (9), 184-192.

Ramió, C. (2014). Manual para los atribulados profesores universitarios. Madrid: Catarata.

Rey-López, J.P. y González, C.A. (2018). Research partnerships between Coca-Cola and health organizations in Spain. European Journal of Public Health, 29 (5), 810-815. doi: https://10.1093/ eurpub/cky175

Rodríguez De Santiago, J.M. (2012). Libertad de investigación científica y sexenios. Revista catalana de dret públic, 44, 225-252.

Rodríguez-Victoriano, J.M. (2017). La praxis de la excelencia universitaria entre la paranoia de sus promotores y la culpa de sus víctimas: hacia la recuperación del deseo docente y la universidad pública. Teknokultura. 14 (1), 85-103.

Saltelli, A. (2017). “La ciencia hoy es una mercancía”. CTXT \& Observatorio Social de la Caixa. [Recuperado de https://goo.gl/F1mqp3]

Sampedro, J. (2017). Logro científico. El País. [Recuperado de https://goo.gl/cEZ9Lf]

Sánchez-Caballero, D. (2017). Los años de recortes dejan las plantillas universitarias envejecidas y precarizadas. Eldiario.es. [Recuperado el 13 de julio de 2017 de https://goo.gl/PSAoGZ]

Serrano Sanz, F.; Holocher-Ertl, T.; Kieslinger, B.; Sanz Garcia, F. y G. Silva, C. (2014). White Paper on Citizen Science in Europe available. [Recuperado de https://cutt.ly/neL3koW].

Serrano, F., Iñiguez, D., Clemente, J., Rivero, A., Ibáñez, M., Ruiz, G., Sanz, F., Ferrer, A., Tarancón, A., Pelacho, M., Guardia, L. y Perla P. (2017). Informe del observatorio de la ciencia ciudadana en España. Madrid: Fundación Ibercivis.

Torres, L.A.; Nuñez, L.A.; Torréns, R. y Barrios, E. (2011). Implementación de un repositorio de datos científicos usando Dspace. Renata, 1(2), 101-117.

Torres-Salinas, D.; Robinson-Garía, N. y Cabezas-Clavijo, A. (2012). Compartir los datos de investigación: introducción al data sharing. El Profesional de la Información, 21(2), 173-184.

ÚLTIMO CERO. (2016). Los científicos se rebelan contra la 'muerte anunciada' de la investigación en Castilla y León. [Recuperado de https://n9.cl/c9nb]

Villareal, A. y Escudero, J. (2018). Todos contra Elsevier, el gigante editorial científico que cobra a España 25 'kilos' al año. El Confidencial. [Recuperado de https://goo.gl/iBkU8K] 